\title{
Design of IoT based fire-watching and Atmospheric Environment Monitoring Systems Applied with Compound Sensors and Image Processing
}

\author{
Seung-su Lee ${ }^{1}$, Woo-chul-Jung ${ }^{2}$, Joon-hoon Park ${ }^{3}$ \\ 1,2 380-702 Dept. of Control \& Instrumentation Eng, Korea National Univ. of \\ Transportation, \#50 Daehak-ro, Chungju-si, Chungbuk, Korea \\ $3\left({ }^{*}\right.$ Corresponding Author) 380-702 Dept. of Control \& Instrumentation Eng, Korea \\ National Univ. of Transportation, \#50 Daehak-ro, Chungju-si, Chungbuk, Korea \\ 1torysu88@ut.ac.kr, ${ }^{2}$ jwc1736@ut.ac.kr, ${ }^{3}$ jhpark@ut.ac.kr
}

\begin{abstract}
Recently, people's sensitivity to industrialization, external fine dust, and real life dust has increased substantially. As a result, the time during which people stay indoors using air cleaners has been gradually increasing. Therefore systems to monitor dust concentrations and atmospheric environments in indoor environments where people reside are necessary. In this respect, in the present study, a system was developed that can monitor comprehensive indoor atmospheric environments by acquiring fine dust concentrations, temperatures, and humidity so that generalusers can easily identify the results. In addition, as kitchen utensils and electrical machinery and apparatuses are used indoors for long periods of time the importance of fire-watching has been increasing. Therefore, in the present study, reat time fire-watching was enabled by installing fire-watching and monifong systems in indoor sections where fire may occur. Dust sensors, temperature and humidity Csensors were used to determine dust concentrations and indoor air quality, Ardumo microcontrollers were used to enable data acquisition and monitoring, and Raspbercy Pi microcontrollers and image sensors were combined to implementfire-watching and monitoring systems.
\end{abstract}

Keywords: atmospheric envinonment monitoring, image processing, fire-watching, dust sensor, micocontwoller

\section{Introduction}

An international organization announced that 4.3 million people die per year due to indoor air pollution resulting from industrialization, inflows of outdoor fine dust, indoor dust, and pollutants generated in the process of cooking in the kitchen. However, whereas resident can easily respond to the pollution of outdoor atmospheric environments because related information can be easily obtained through mobiles and broadcasting, they cannot easily recognize and respond to the pollution of indoor air environments because elements for determination of indoor air quality are not diverse. Therefore, in the present study, a system was developed that enables the acquisition of indoor atmospheric environment, temperature, and humidity data using Arduino board based gas sensors, dust measuring sensors, temperature and humidity sensors and enables direct gas leakage detection and fire-watching and monitoring using Arduino boards, short-distance fire detection sensor modules, gas sensor modules, and camera modules. That is, a comprehensive home monitoring system was designed that can be applied to home environments and diverse indoor environments for atmospheric environment monitoring and fire-watching as it enables monitoring of indoor atmospheric environments through compound sensors and accurate fire-watching monitoring through image processing and 
fire detection sensors. Diverse compound sensors were used to check atmospheric environments and Internet access was enabled through the mainboard of the fire-watching and monitoring system. The transmission of the dust concentration and atmospheric environment information measured through the foregoing and the transmission of fire images to the outside were also enabled so that IoT based fire-watching and home monitoring systems can be implemented.

\section{Monitoring System Hardware Implementation}

\subsection{Compound Sensor Data Processing Unit Implementation}

The home monitoring system consists of a fire-watching and monitoring system for fire-watching and an indoor atmospheric environment monitoring system. Each monitoring system was implemented by fusing compound sensors using an Arduine board as a mainboard. The fire-watching and monitoring system was implemented using shortdistance fire-watching sensors, gas leakage detection sensors, and temperature/humidity sensors and the indoor atmospheric environment monitoring system was implemented using dust sensors and temperature/humidity sensors. Figurel below show the firewatching and monitoring system and Figure 2 below shows the indoor/atmospheric environment monitoring system [1].

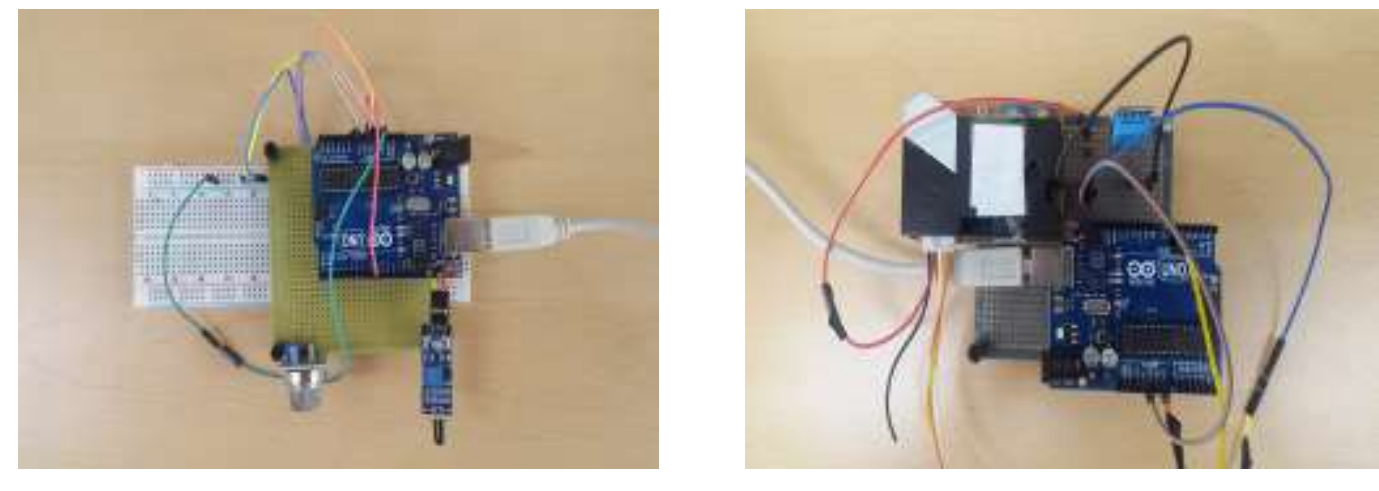

Figure 1. Fire watching System . Fire-watching System

Figure 2. Indoor Atmospheric Environment Monitoring System

\subsection{Sensor configuration}

\subsubsection{Short-distance Fire Detection Sensor}

The NFFDSM from NEOTICS Co. was used as a short-distance fire detection sensor module in the fire-watching monitoring system. This sensor module is used for shortdistance fire, flame, spark detection, can detect flames with wavelengths in a range of $760-1100 \mathrm{~nm}$ at distances up to $50 \sim 80 \mathrm{Cm}$, and is attached with a power LED and a detection output LED for easy judgment by the user. Its operating power is characterized by a wide range of working voltage, which is DC $3.3 \mathrm{~V} \sim \mathrm{DC} 5 \mathrm{~V}$ and its detection outputs are at the TTL level so that they can be directly used by the MCU. Table 1 below shows the characteristics of the short-distance fire detection sensor [2]. 
Table1. Specification of the Short-distance Fire Detection Sensor

\begin{tabular}{|c|c|}
\hline Specification & Detail \\
\hline Power voltage & DC 3.3 5V \\
\hline Operational current & $10 \mathrm{~mA}$ \\
\hline Detection output & $\begin{array}{c}\text { At normal times 'H' } \\
\text { When fire has been detected 'L' }\end{array}$ \\
\hline
\end{tabular}

\subsubsection{Temperature/humidity Sensor}

Temperature/humidity sensors were used in both the fire detection monitoring system and the indoor atmospheric environment monitoring system. Since the fire detection monitoring system is used in the vicinity of electronic equipment or kitchen utensils, temperature information should be monitored using temperature/humidity sensors. In addition, temperature/humidity data were used in the indoor atmospheric environment monitoring system to acquire indoor environment information. DHT11 sensors were used as temperature/humidity sensors in both systems. The DHT11 sensor outputs digital signals, operates with the power of $+5 \mathrm{~V}$, has a temperature measuring range of $0 \sim 50^{\circ} \mathrm{O}$ (measurement error $\pm 5^{\circ} \mathrm{C}$ ) and a humidity measuring range of 20 90\%(measurement error $\pm 5 \%$ )[3].

\subsubsection{Gas Detection Sensor}

In the present study, MQ-6 gas detection sensor modules were used in the firewatching and monitoring system, MQ-6 gas delection sensors are sensitive to propane gas, butane gas, LPG, and natural gas and can measure the concentrations. When any of the gases has been detected, the value of the internal resistance of the MQ-6 gas detection sensor is changed so that the concentration of the gas can be measured by receiving the resultant dutput voltage of the sensors. In addition, the sensitivity of this gas sensor to certain gases can be adjusted by connecting a variable resistor to the output terminal of the gas sensor. Therefore, MQ-6 gas sensors can be connected to the fire-watching and monitoring system to monitor the leakage of butane gas and LPG gas that are mainly used at home or restaurants and can quickl detect and preyent the ignition and secondary damage. Figure 3 shows a gas sensor application circuit and Figure 4 shows the sensitivity of the sensor to certain gasses accopong to the ratio of sensor resistance to variable resistance [4].

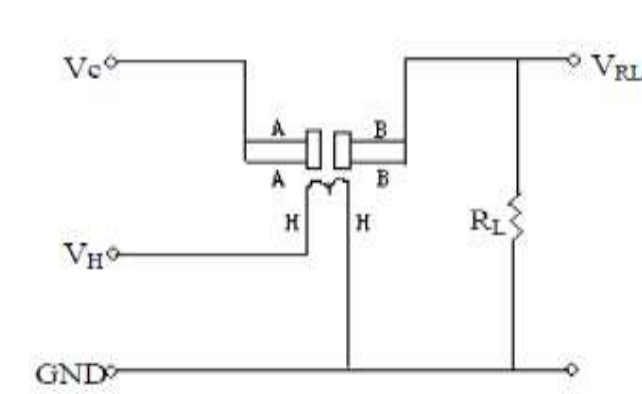

Figure3. MQ-6 Sensor Circuit

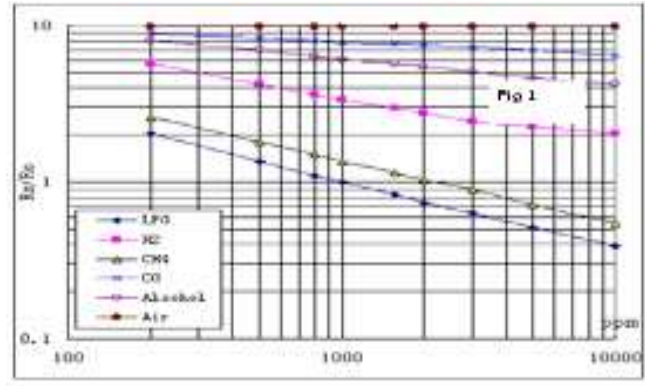

Figure4.Sensor Sensitivity Levels by Type of Gases 


\subsubsection{Dust Sensor}

DSM501 dust sensors from SAMYOUNG Co. were used in the indoor atmospheric environment monitoring system. These sensors are PWM output type dust detection sensors that can measure lum (micrometer) or larger dust. These sensors operate with $5 \mathrm{~V}$ power, can be easily connected to the Arduino used as the mainboard, and can detect tobacco fumes, household dust, pollen, and fine dust. The Each DSM501 module consists of a heater, a dust detection unit, a signal amplification unit, and a signal output unit. When dust particles have been detected, the PWM output is changed into LOW. The dust concentration can be measured by measuring output signals for $30 \mathrm{sec}$. and adding up LOW output times during the 30 sec. Figure 5 below shows the ratios of LOW output signals and related dust concentrations and Figure6 shows the method of calculating dust concentration through the measurement of LOW output signals [5].
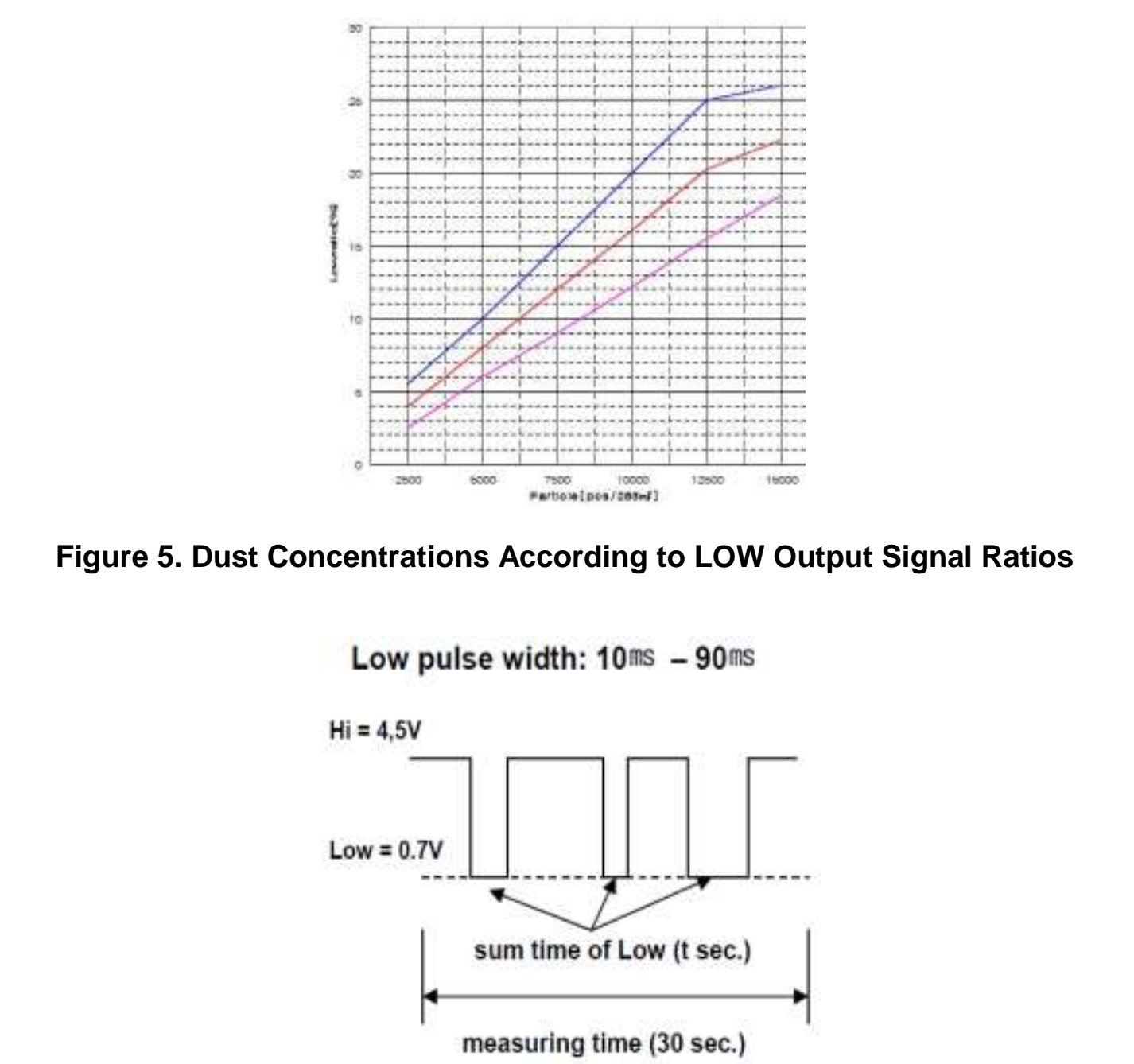

Low ratio $[\%]=t(\mathrm{sec}) / 30(\mathrm{sec})^{*} 100$

Figure 6. Method of Measuring LOW Output Signal Ratios 


\subsection{Implementation of the Image Data Processing Unit}

In the present study, image data processing units were used for fire-watching and monitoring and household environment monitoring. Raspberry Pi microcontrollers were used as the main boards of the image data processing units [6]. Based on 32BIT ARM Cortex-A7 core processors, Raspberry Pi microcontrollers support diverse peripheral systems such as USB ports and wire/wireless Internet. In addition, they can be easily connected through the web because they loaded with an operating system. Camera modules were connected to Raspberry Pi main boards to apply monitoring for fire detection and to enable indoor environment monitoring. Figure 7 below shows the image data processing unit configured in the present study. the camera module is connected to the CSI (Camera Serial Interface) terminal in the mainboard of the fire-watching and monitoring system and was installed with a CMOS image sensor from Omni vision Co. It is characterized by small size, can take 5Mega Pixel still cuts, and supports 1080p, 720p, and 640x480p video filming modes [7].

\section{Implementation of Home-monitoring System Software}

\subsection{Fire detection Algorithm}

The fire-watching and monitoring system implemented in the present study was installe indoors such as houses and restaurants where fire-watching and monitoring is necessary to enable responses to the occurrence of fire by monitoring gas leakage and use of fire for long periods of time that can increase the possibility of fire. The fire detection monitoring system first judges whether fire has been detected based on gas leakage detection sensors, short-distance fire detection sensors, and temperature/humidity sensors. When fire has been detected, it obtains images using the image data processing unit and finally judges whether fire has occurred. Figure 8 below shows the operating algorithm of the fire-watching and monitoring system. 


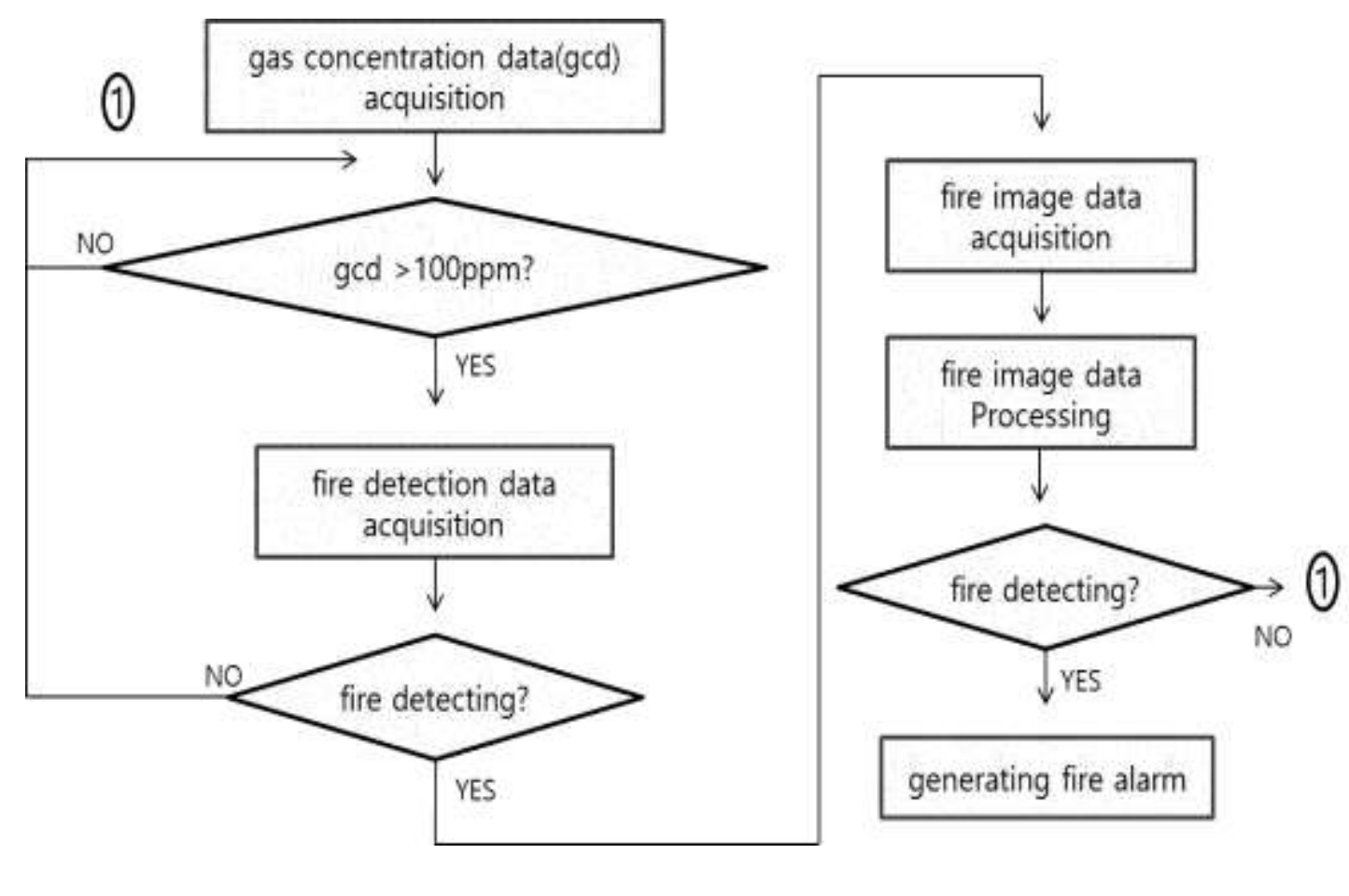

Figure 8. Fire-watching and Monitoring Algorithm

\subsection{Integrated Indoor Environment Information Acquisition Algorithm}

In the present study, an indoor atmospheric environment monitoring system was used to obtain real time infomation on indoor atmospheric environments and atmospheric environment alarm levels were set to enable the recognition of integrated indoor environments. First real lime indoor temperature/humidity data are obtained to judge information on indoor temperatures and humidity and indoor dust concentrations are obtained from the dust sensor to judge comprehensive indoor dust coneentrations due to factors such as fine dust, tobacco fumes, and yellow sand so that information on integrated indoor atmospheric environments can be recognized. Figure9 below shows the indoor atmospheric environment monitoring algorithm. The obtained temperature/humidity and dust concentrations are analyzed and indoo environment comfort levels are outputted to the LCD module so that the user can recognize the current indoor environment comfort state. According to Korea Environment Corporation's fine dust related data, a fine dust forecast system is operated currently and forecast levels are divided into five levels as shown in Table 2 below considering atmospheric environment standards and health mpacts [8]. Based on the forecast levels, indoor temperature/humidity information is delivered and fine dust concentrations divided into three levels; good (eve 1), moderate (level 2), and bad (level 3) are outputted so that residents can recognize indoor fine dust concentration levels.

Table 2. Fine Dust Forecast Levels

\begin{tabular}{|c|c|c|c|c|c|}
\hline Section & Good & Moderate & A little bad & Bad & Very bad \\
\hline $\begin{array}{c}\text { Concentration } \\
(\mu \mathrm{g} / \mathrm{m} 3)\end{array}$ & $0 \sim 30$ & $31 \sim 80$ & $81 \sim 120$ & $121 \sim 200$ & $201 \sim$ \\
\hline
\end{tabular}




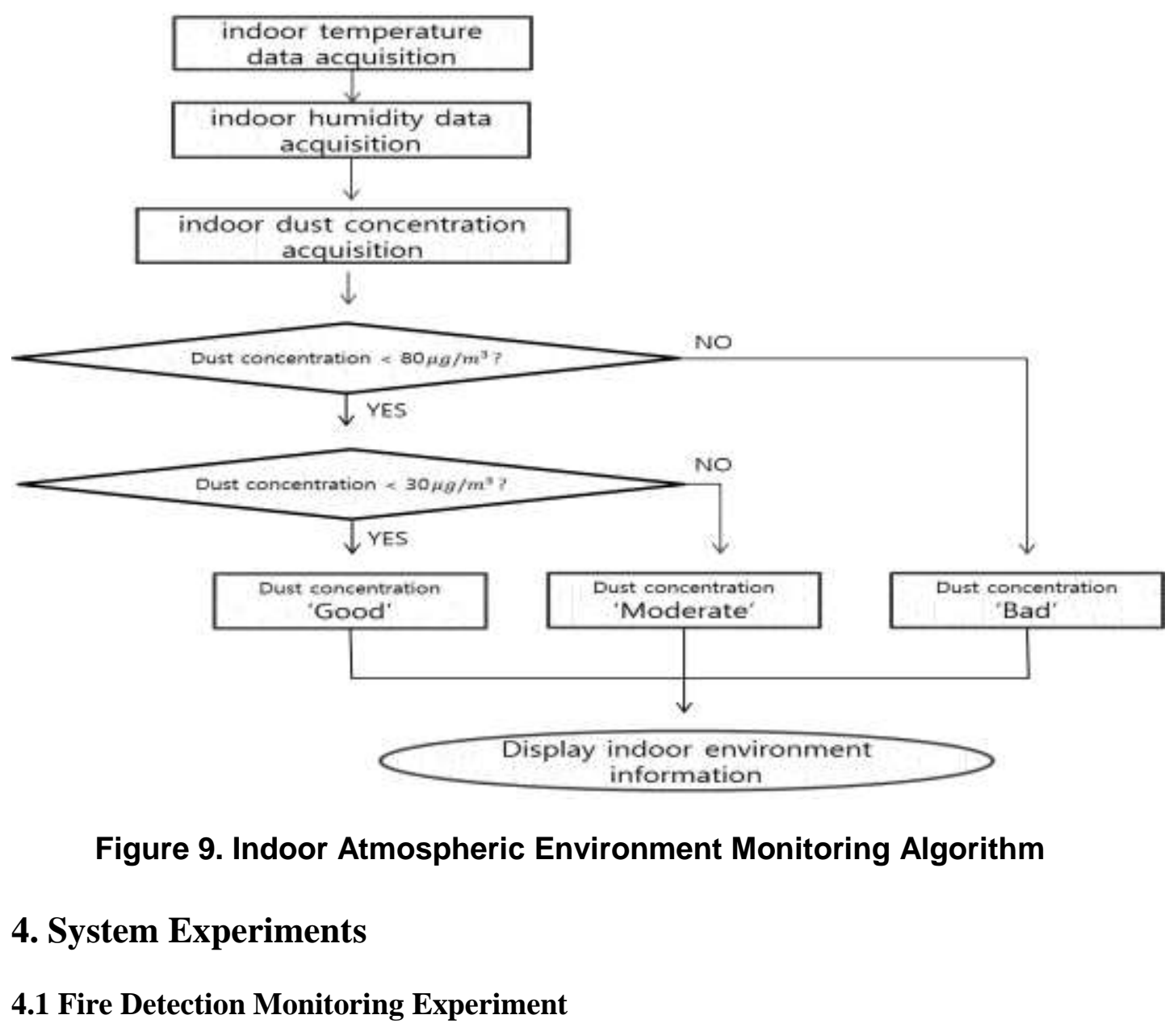

To conduct fire detection monitoring experiments, a small quantity of butane gas was leaked in a closed space and the gas leakage was detected and sparks and flames were detected through the ignition of portable lighters. The experiment was conducted in thefollowing order First, whether fire has occurred is judged based on the output of the short-distance fire detection sensor. The short-distance fire detection sensor generates HIGH signals when no fire has been detected and generates LOW signal when any flame or spark has been detected. Second, whether any gas has been leaked is detected using an MQ-6 sensor. Table 3 below shows the results of monitormg of whether any flame or spark has been detected and the concentration of leaked gases by analyzing the data obtained from the sensor and Figure 10 shows the source codes of the fire-watching and gas concentration acquisition program. As can be seen in Table 3, when fire has been set, the firewatching sensor generates LOW signals to display the fire detection. In addition, the analog voltage output by the gas detection sensor is converted into digital values to judge whether the gas has been leaked through the ADC process of the Arduino mainboard. The A/D converted sensor output voltage in closed spaces where no gas has been leaked is 70. As can be seen in Table 3, the sensor output voltage (gas concentration) exceeded 70 after the gas was leaked and increased in proportion to the exposure time. The fact that fire has been detected can be identified by analyzing the data obtained through the sensor module and gas sensor. 


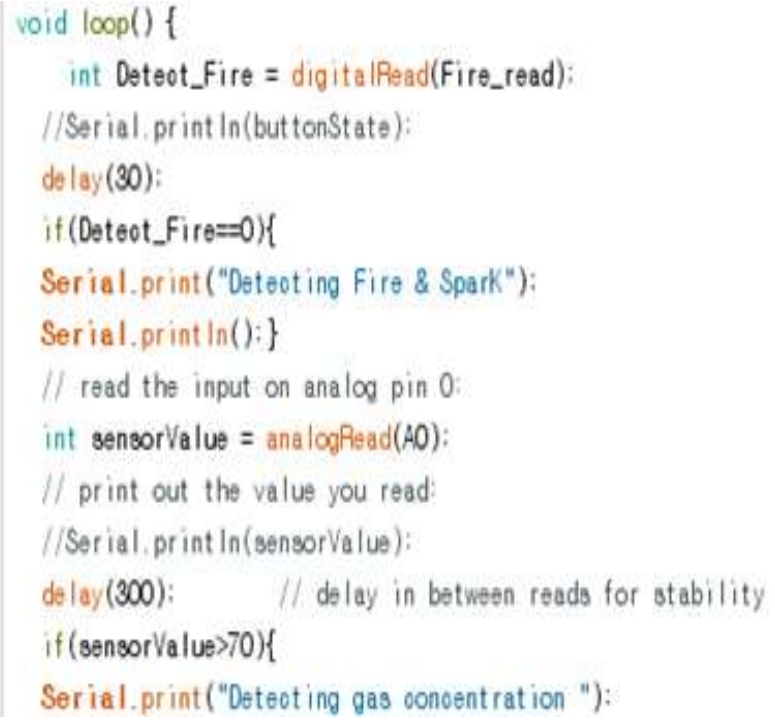

Figure 10. Fire-watching and Gas Concentration Acquisition Program

Table 3. Real Time Fire-watching and Gas Concentration

\begin{tabular}{|c|c|c|}
\hline Number of times & $\begin{array}{c}\text { Real time as } \\
\text { concentration(p) }\end{array}$ & $\begin{array}{c}\text { Whether any spark or fire has } \\
\text { been detected }\end{array}$ \\
\hline 1 & 93 & $\mathrm{O}$ \\
\hline 2 & 171 & $\mathrm{O}$ \\
\hline 3 & 310 & $\mathrm{O}$ \\
\hline 4 & 591 & $\mathrm{O}$ \\
\hline
\end{tabular}

However, in the case of sensor based fire detection, accurate sizes or states of flames cannot be easily icentified. In the present study, to overcome this shortcoming, the system was installed in actual regions where fire may occur to obtain real time image data using an image data processing unit. In addition, an image processing program was applied to additionally check whether actually fire has been set so that 2 accurate fire detection algorithm can be applied in addition to the fire detection sensor and gas sensor. Figure 11 below shows the image data obtained through the image data processing unit to conduct fire-watching and monitoring experiments. Fire images were induced to form experimental environments. After recognizing ignition through the fire detection sensor and gas concentration measuring sensor, actual ignition images were taken through the mage processing unit to judge the size of flames and whether fire had been set. Two image processing techniques were used to conduct fire region detection experiments. First, to detect only fire regions out of the background region, the difference image technique was applied to separate the background region and the fire regions from each other. When the difference image technique is applied, accurate fire detection is possible regardless of external environments. Thereafter, the bainalization technique was applied to the derived fire images to enable accurate detection of fire regions regardless of the sizes of fire ranging from micro fire to large fire. Figure11a shows the background image before the fire was set, Figure11-b shows the image after the fire was set and Figure11-c shows the difference image in which only the fire region was derived. 


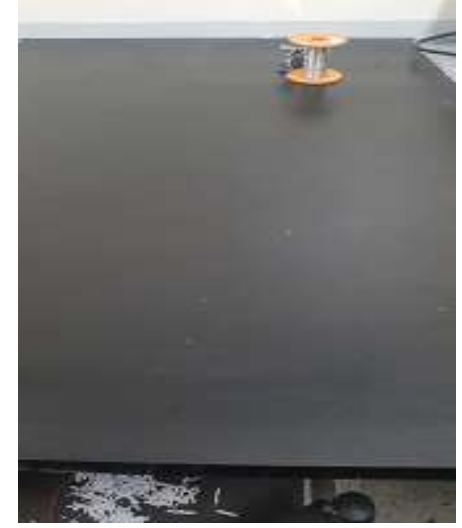

(a)

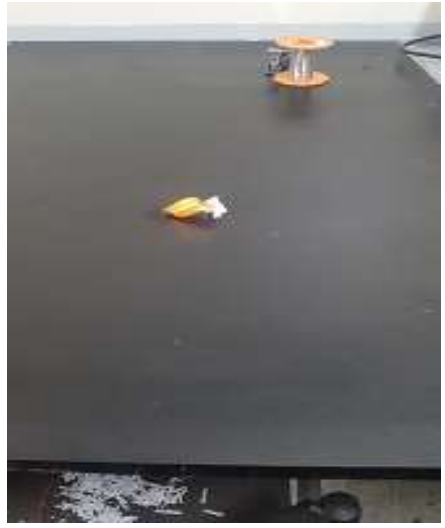

(b)

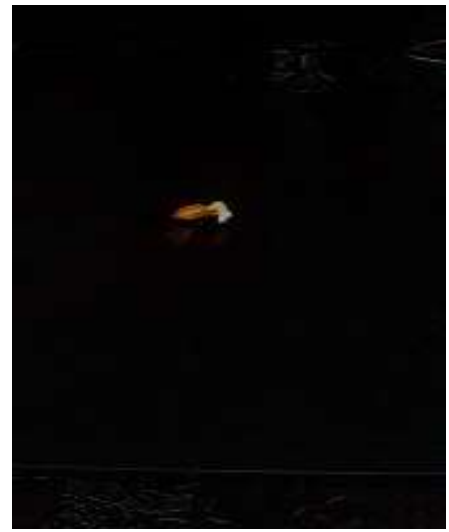

(c)

Figure11. Fire Detection Image

no fire is detected in the background image in Figure 11-a. The fire image in Figure 11-b was obtained after setting fire and the difference image technique as applied to the image to obtain the accurate fire region only in Figure 11-c. Through the process shown above, fire-watching monitoring systems can be installed in the vieinity of kitchen utensils and home appliances to detect fire images. In addition, the size of fire can be estimated by analyzing the derived difference images for accurate responses according to the sizes of fire. The histogram of the derived difference îmage can be analyzed to detect accurate sizes and regions of fire. The his ogram means the unique brightness values of pixels in the image. The difference amages are binories and threshold values are designated based on the brightness alues of pixels to numerically analyze whether fire has been set and the sizes of five Figure 12 shows a binary image derived after conducting bainalization with a threshold value of 87 . As can be seen from the image, accurate and clearer separation of fire regions, is possible. The sizes of fire regions can be identified using the pixelbrightness value data. This bainalization technique will enable accurate detection of fire regions if it is applied after identifying the level of illumination in the environment where the fire-watching and monitoring system has been installed and setting the optimam threshold value that fits the level of illumination.

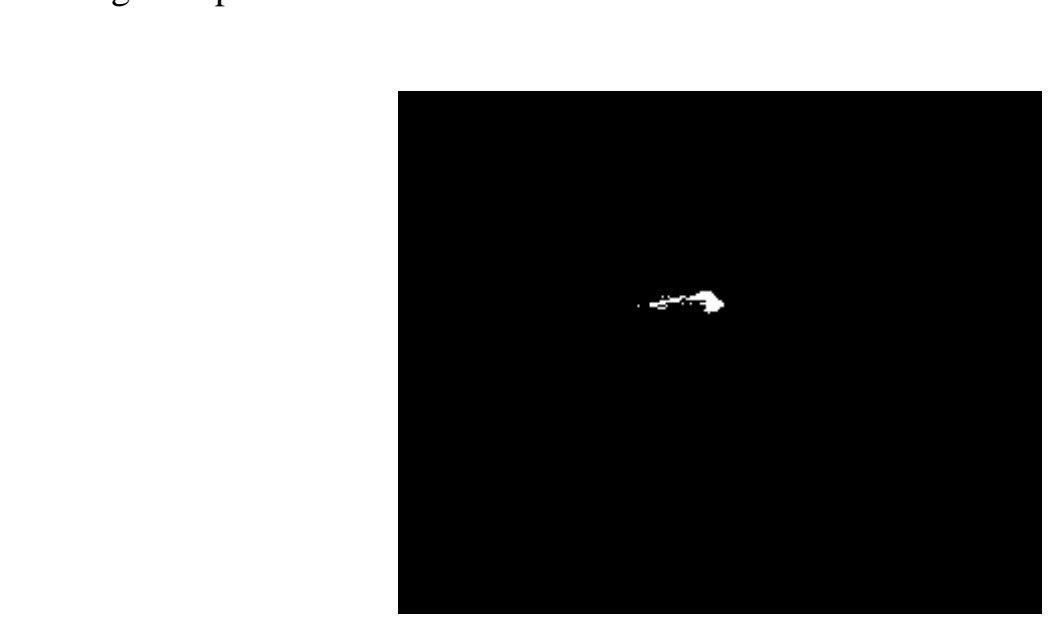

Figure 12. Binalization of Detected Fire Image 
In addition, the bainalization technique was applied to detect larger fire regions. Figure 13- a below shows the difference image of the fire region and Figure 13-b shows the binary image of the fire region. As can be seen in the Figure, fire can be detected accurately regardless of the sizes of fire. In addition, fire sizes can be estimated by measuring the sizes of fire regions in the image. Through the foregoing, fire information can be analyzed for all sizes of fire ranging from micro-fire to large fire and responses can be made by the level of fire.

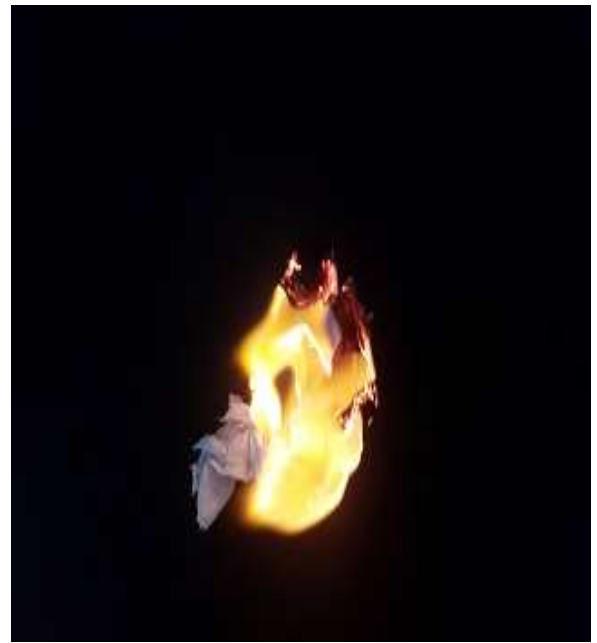

(a)

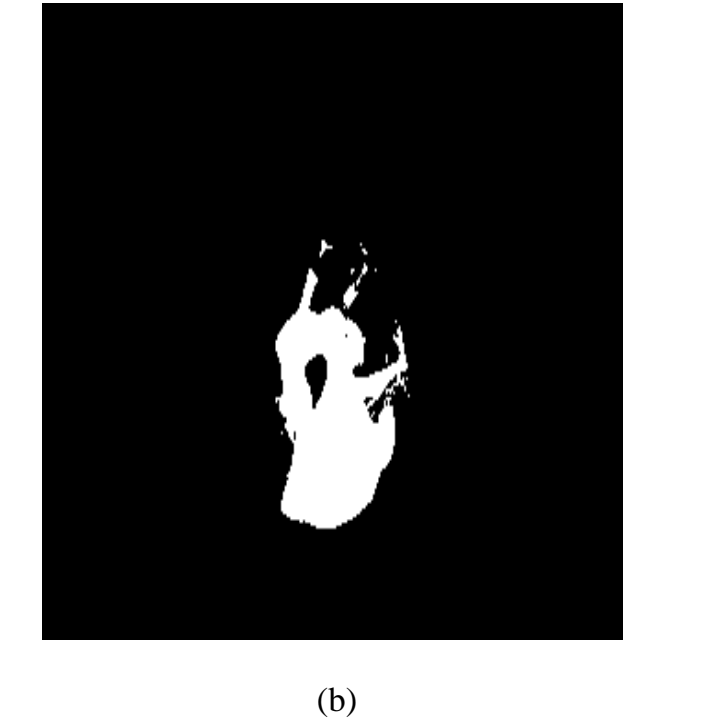

(b)

Figure 13. Original Fire Image and Binarized Image

\subsection{Indoor Atmospheric Environment Monitoring Experiment}

The indoor atmospheric environmen monitoring system obtains real time temperature/humidity information to monitor indoor humidity and temperature conditions and detect (1ndoor dust concentrations. The dust sensor used in the present study can detect even $1 \mu \mathrm{m}$ (micrometer) sized fine dust and harmful smoke. Tobacco fumes were generated in a closed space to form a harmful environment for dust concentration measuring experiments and changes in dust concentrations were experimented by ventilating the space during exposure to tobacco fumes for accurate measurement of dust concentrations. Indoor dust concentrations before releasing tobacco furmes, after releasing tobacco fumes, and after ventilation were compared with each other to conduct comprehensive indoor atmospheric environment monitoring experiments [9].Table 4 below shows temperature/humidity data and changes in dust concentrations for $30 \mathrm{sec}$. before and after exposure to the harmfulenvironment. As can be seen in Table 3, the dust concentration was low for the (1rs) $30 \mathrm{sec}$. because there was only indoor residual dust. However, the dust concentration increased rapidly thereafter due to the inflow of tobacco fumes. The space was ventilated before 1 minute and 30 second passed and the experiment was continued. The dust concentration gradually decreased thereafter to the level during the first $30 \mathrm{sec}$. The space was closed for 1 minute from 2 minutes after beginning of the experiment and large amounts of tobacco fumes were released again. As a result, the dust concentration increased rapidly. 
Table 4. Temperature/humidity Data and Real Time Changes In the Dust Concentration

\begin{tabular}{|c|c|c|c|}
\hline $\begin{array}{c}\text { Number of times } \\
\text { (interval: } 30 \mathrm{~s})\end{array}$ & $\begin{array}{c}\text { Dust concentration } \\
(\mu \mathrm{g} / \mathrm{m} 3)\end{array}$ & $\begin{array}{c}\text { Temperature } \\
\left({ }^{\circ} \mathrm{C}\right)\end{array}$ & $\begin{array}{c}\text { Humidity } \\
(\%)\end{array}$ \\
\hline 1 & 15 & 26 & 41 \\
\hline 2 & 158.9 & 26.5 & 41 \\
\hline 3 & 109.2 & 24.5 & 42 \\
\hline 4 & 30.8 & 24 & 40 \\
\hline 5 & 305.5 & 24 & 41 \\
\hline 6 & 164.6 & 25.5 & 41 \\
\hline
\end{tabular}

Meanwhile, the temperature/humidity output of the indoor atmospheric environment monitoring system for the experiment environment and the temperature/humidity output for fine dust concentration levels are shown in Figure 14 and Figure 15 below respectively. Figure14 shows the values in the experiment 1 in Table 4 and Fure15 shows the value in the experiment 2 . As can be seen in the Figures, the system enabled the user to recognize indoor temperature/humidity conditions as well as the ranks of risks of dust concentration thanks to the difference in dust concentrations before and after smoking.

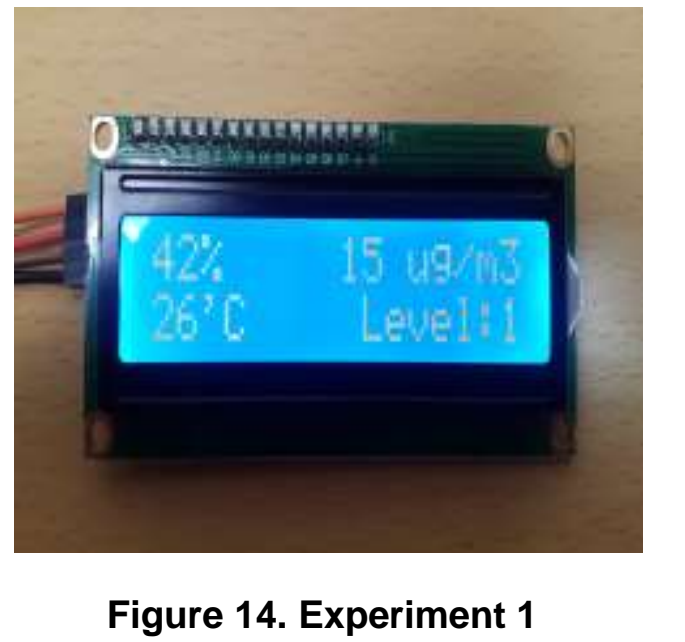

\section{Conclusion}

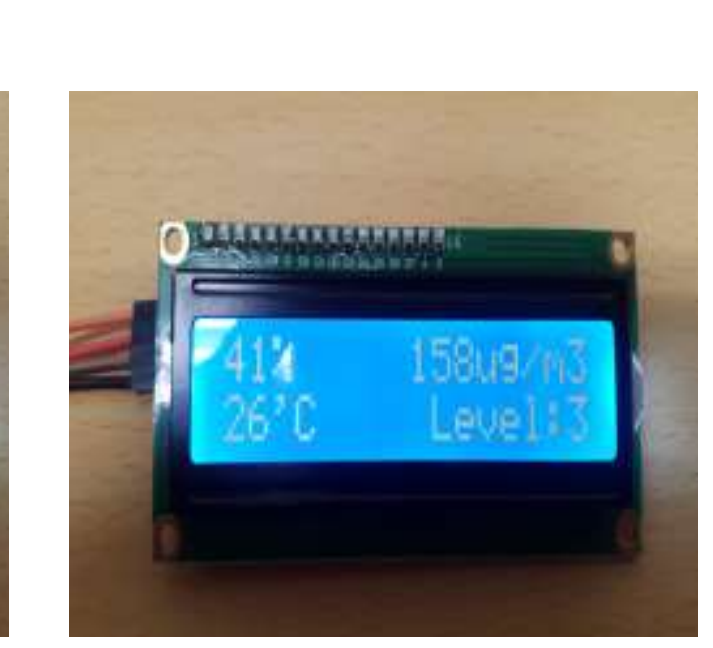

Figure 15. Experiment 2

In this papen both indoor atmospheric environment monitoring and indoor firewatching monitoring were enabled using image processing and compound sensors. Among elements that affect indoor atmospheric environments, real time indoor temperature/humidity data were obtained through Arduino board based temperature and humidity sensors to judge information on indoor temperature and humidity and indoor dust concentrations were obtained from the dust sensor to judge comprehensive indoor dust concentrations due to factors such as fine dust, indoor residual dust, tobacco fumes, and yellow sand. In addition, the user was enabled to for comfortable indoor environments by immediately responding to any problem exceeding appropriate levels through ventilation or using air cleaning products through monitoring of accumulated data and real time fire-watching and monitoring was enabled using Arduino boards, shortdistance fire detection sensor modules, gas sensors, Raspberry Pi mainboards, and camera modules. The user was also enabled to conduct direct gas leakage detection and fire-watching and monitoring by obtaining fire images when fire has been detected after first judging whether fire has occurred through fire detection sensors. In addition, the user 
was also enabled to identify flame sizes for all fires ranging from micro-fire to large fire. Users are expected to be able to prevent damage to human lives and properties due to large fire through the system. In addition, since the system was identified as being capable of indoor atmospheric environment monitoring and fire-watching and monitoring, it was confirmed that the system can be it installed and used in many places such as home environments, restaurants, and public places depending on dust concentrations and sensitivity to fire. Future tasks are supplementing the system so that immediate actions can be taken through linkage with fire authorities through communication when fire is not extinguished and increasing the utility of the system by grafting measures to respond to invasion from the outside through image processing.

\section{Acknowledgments}

"This research was financially supported by the Ministry of Education (MOE) and National Research Foundation of Korea (NRF) through the Human Resource Training Project for Regional Innovation (No. 2014H1C1A1066414)."

"This was supported by Korea National University of Transportation in 2016.'

\section{References}

[1] L.sueng-su and J.woo-chul and P.joon-hoon, "Implementation of an IoT based indoor harmful environment monitoring system", Asia-pacific Proceedings of Applied Science and Engineering for Better Human Life, Jeju, Korea, (2016) August 16-19

[2] NS-FDSM-K datasheet

[3] http://www.micropik.com/PDF/dht11.pdf

[4] http://www.probots.co.in/Datasheets/MQ6.pdi

[5] 3-1 Specification DSM501.pdf, DSM501 datasheet

[6] J. woo-chul and L. sueng-su and P. joon-hoon, "Design of optimal snowmelting system with snowfall image processing based on the Ior technology", Asia-pacific Journal of Multimedia Services Convergent with Art, Humanities, and Sociology, Eol 5, no.6, (2015), pp. 521-530

[7] https://www.raspberrypi.org/documentation/hardware/camera.md

[8] Fine dust brochure, Korea Environment Corporation

[9] https://github.com/empiere/ayduino/blob/master/Dust sensor_SamYoung_DSM501.ino

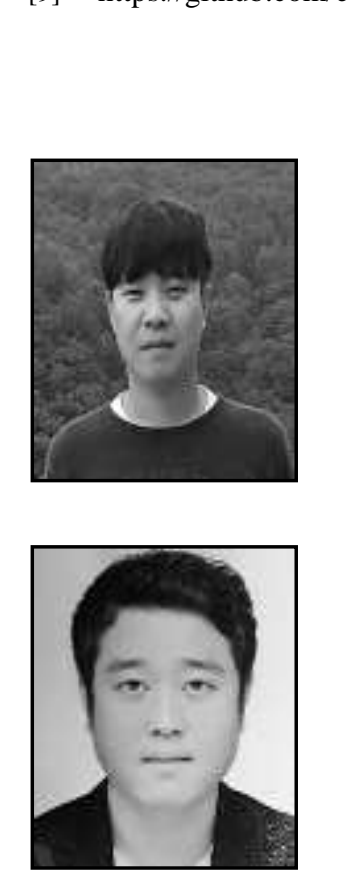

\section{Authors}

\section{Seung-Su-Lee,}

Gracuate student of Korea National University of Transportation Major is Control and instrumentation Eng.

Graduated of Korea National University of Transportation

A field of research is embedded system and IoT

\section{Woo-Chul-Jung,}

Graduate student of Korea National University of Transportation

Major is Control and instrumentation Eng.

Graduated of Korea National University of Transportation

A field of research is embedded system and IoT 


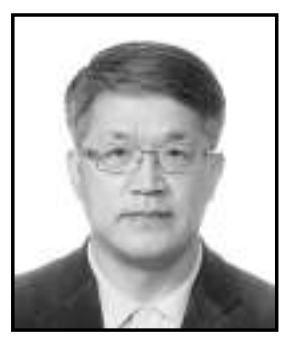

Joon-Hoon Park received the B.S. degree in electrical engineering from SungKyunKwan University in 1981 and the M.S. and the Ph.D. degree in control engineering from SKK Univ., in 1983 and 1992, respectively. He was a senior researcher of the R\&D Center, Samsung Electronics Co. from 1982 to 1990. And he was a Post-Doc. Fellow of the Leicester University in UK and the University of Western Ontario, Canada. Since 1991, he is currently professor of the Department of Control and Instrumentation Eng., Korea National University of Transportation. He is interested in optimal control theory and applications

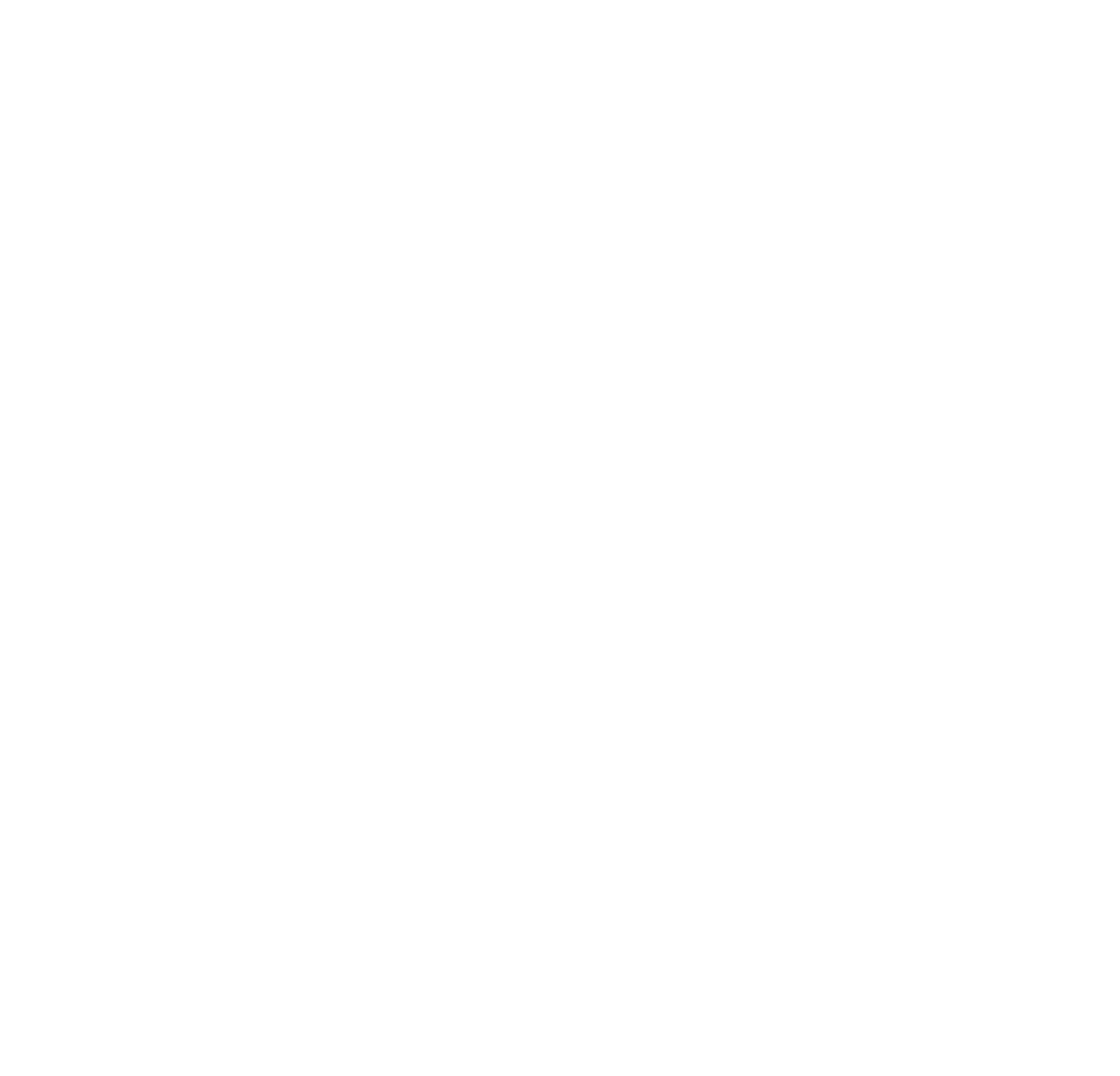


International Journal of Smart Home

Vol.10, No.12 (2016)

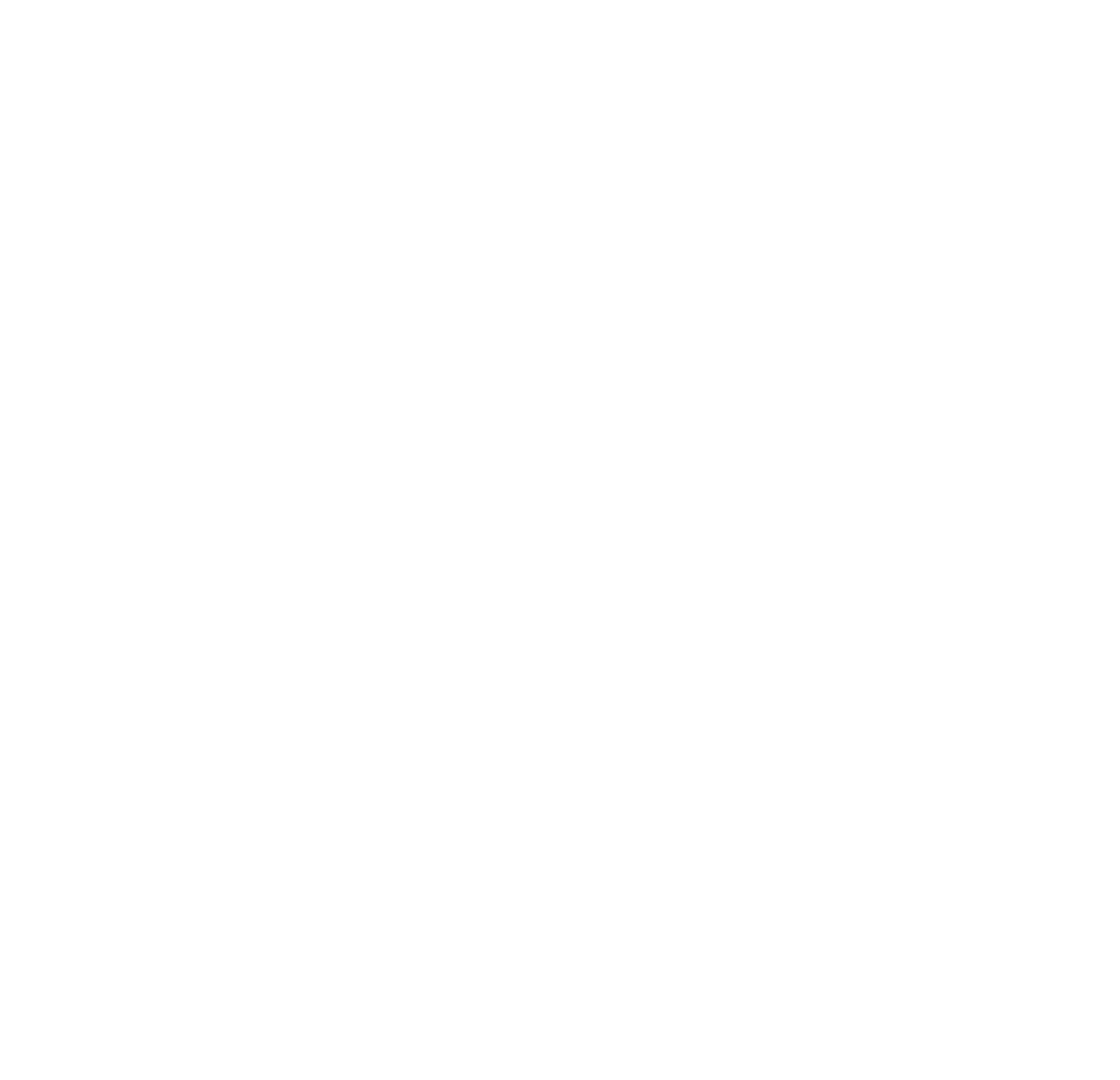

